\title{
Attractor-repeller pair of topological zero modes in a nonlinear quantum walk
}

\author{
Y. Gerasimenko, ${ }^{1,2}$ B. Tarasinski, ${ }^{1}$ and C. W. J. Beenakker ${ }^{1}$ \\ ${ }^{1}$ Instituut-Lorentz, Universiteit Leiden, P.O. Box 9506, 2300 RA Leiden, The Netherlands \\ ${ }^{2}$ Department of Physics, Taras Shevchenko National University of Kyiv, Volodymyrska Street 64/13, Kyiv 01601, Ukraine
}

(Received 8 December 2015; published 23 February 2016)

\begin{abstract}
The quantum-mechanical counterpart of a classical random walk offers a rich dynamics that has recently been shown to include topologically protected bound states (zero modes) at boundaries or domain walls. Here we show that a topological zero mode may acquire a dynamical role in the presence of nonlinearities. We consider a one-dimensional discrete-time quantum walk that combines zero modes with a particle-conserving nonlinear relaxation mechanism. The presence of both particle-hole and chiral symmetry converts two zero modes of opposite chirality into an attractor-repeller pair of the nonlinear dynamics. This makes it possible to steer the walker towards a domain wall and trap it there.
\end{abstract}

DOI: 10.1103/PhysRevA.93.022329

\section{INTRODUCTION}

A classical random walk is invariably associated with diffusive motion, but quantum superposition and interference allow for a more varied dynamics. A quantum walk can explore phase space more rapidly than its classical counterpart [1-3], a shift from diffusive to ballistic dynamics that is at the origin of the quadratic speedup of quantum search algorithms [4,5]. Diffusion is recovered for temporal disorder, while spatial disorder can induce an Anderson quantum phase transition to localized wave functions [6-12].

Two recent developments have further enriched the phenomenology. One development is the discovery that quantum walks can exhibit a topological phase transition, at which a bound state (a so-called zero mode) appears at a boundary or domain wall [13-21]. A second development involves the introduction of nonlinearities in the dynamics [22,23]. These have been associated with soliton structures $[24,25]$ and investigated as a means to speed up the quantum search [26]. Here we wish to connect these two separate developments, and explore how nonlinearities manifest themselves in a topological quantum walk.

We consider the simplest case of a one-dimensional discrete-time quantum walk in the chiral orthogonal symmetry class (also known as class BDI, familiar from the Su-Schrieffer-Heeger model [27]). The topological phase transition manifests itself by the appearance of a pair of zero modes of opposite chirality. We demonstrate that these zero modes may survive in the presence of nonlinearities and moreover acquire a special role as the attractor and repeller of the nonlinear dynamics.

\section{FORMULATION OF THE LINEAR QUANTUM WALK}

We study the one-dimensional dynamics of a two-level system, represented by a spin- $\frac{1}{2}$ degree of freedom on the lattice $x \in \mathbb{Z}$. We employ a stroboscopic description, so that time $t \in \mathbb{Z}$ is discretized as well as space. The linear dynamics is obtained by repeated applications of a unitary operator $U$ on a spinor $\psi$,

$$
\psi_{t}=(U)^{t} \psi_{0}, \quad \psi_{t}(x)=(u(x, t), v(x, t)) .
$$

Quite generally, a single time step of such a discrete-time quantum walk can be decomposed into two operations: a rotation $R_{\vartheta}$ of the spinor and a shift $S$ to the left or to the right dependent on the spin component:

$$
\begin{aligned}
& R_{\vartheta} \psi=e^{-i \vartheta \sigma_{y}} \psi=(u \cos \vartheta-v \sin \vartheta, u \sin \vartheta+v \cos \vartheta), \\
& S(u(x, t), v(x, t))=(u(x-1, t), v(x+1, t)) .
\end{aligned}
$$

We can combine the two operations as $S R_{\vartheta}$ or $R_{\vartheta} S$, but we prefer to take the symmetrized product [28],

$$
U=R_{\vartheta / 2} S R_{\vartheta / 2} \text {. }
$$

The evolution operator (3) is representative of a chiral orthogonal quantum walk, meaning that $U=U^{*}$ is real orthogonal (particle-hole symmetry) and $\left(\sigma_{x} U\right)^{2}=1$ (chiral symmetry). This BDI symmetry class supports a topologically protected zero mode bound to a domain wall where $\vartheta(x)$ changes sign. Its time-independent state $\Psi_{ \pm}(x)$ satisfies [29]

$$
U \Psi_{ \pm}=\Psi_{ \pm}, \quad \sigma_{x} \Psi_{ \pm}= \pm \Psi_{ \pm} .
$$

The eigenvalue \pm 1 of the Pauli matrix $\sigma_{x}$ distinguishes the chirality of the zero mode [30].

\section{INTRODUCTION OF A NONLINEARITY}

We now introduce a nonlinearity (strength $\kappa$ ) into the quantum walk by inserting a $\psi$-dependent rotation at each time step,

$$
\begin{aligned}
\psi_{t+1}(x) & =U \bar{\psi}_{t}(x), \\
\bar{\psi}_{t}(x) & =\exp \left(-i \kappa M_{z}(x, t) \sigma_{y}\right) \psi_{t}(x), \\
M_{z}(x, t) & =\psi_{t}^{\dagger}(x) \sigma_{z} \psi_{t}(x)=|u(x, t)|^{2}-|v(x, t)|^{2} .
\end{aligned}
$$

This nonlinear time evolution conserves particle-hole symmetry (a real $\psi$ remains real), but chiral symmetry no longer applies. Still, a zero mode $\Psi_{ \pm}$of the linear problem $(\kappa=0)$ remains a stationary state when we switch on the nonlinearity, because $M_{z}=0$ for any eigenstate of $\sigma_{x}$.

To appreciate the new features introduced by the nonlinearity, it is helpful to look at a uniform $\vartheta$ and a real initial state $\psi=(\cos \alpha, \sin \alpha)$ without any spatial dependence. In one time step the angle $\alpha$ is mapped to $\alpha+\vartheta+\kappa \cos 2 \alpha$. This map is invertible if $|\kappa| \leqslant 1 / 2$, but it is not area preserving. 


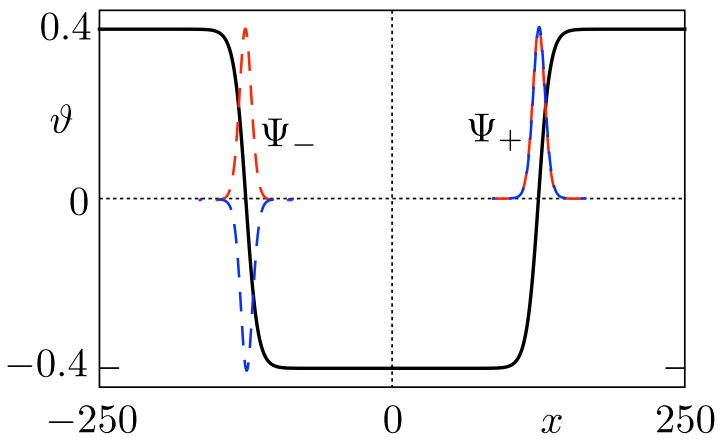

FIG. 1. Solid curve: position-dependent rotation angle $\vartheta(x)$ with a pair of domain walls at which the angle changes sign. Plotted is the profile (6) with $L=500, \lambda=10$, and $\vartheta_{0}=0.4$ used in the numerical simulations. Dashed curves: the two (unnormalized) spinor components of the zero modes bound to the two domain walls, calculated from Eq. (9). The state $\Psi_{ \pm}$is an eigenvector of $\sigma_{x}$ with eigenvalue \pm 1 .

The phase space contracts around one of two attractive fixed points, defined by $\cos 2 \alpha_{c}=-\vartheta / \kappa, \sin 2 \alpha_{c}>0$. Note that this relaxation does not involve any loss of particles: $\sum_{x}\left(|u|^{2}+|v|^{2}\right)$ is conserved by the nonlinear dynamics.

As we will now show, for a spatially dependent $\vartheta(x)$ the zero mode at a domain wall becomes an attractive or repulsive fixed point, depending on its chirality. We first present numerical evidence and then give the analytical solution in the continuum limit.

\section{COLLAPSE ONTO A ZERO MODE}

We take a lattice of length $L$ with periodic boundary conditions, $-L / 2<x<L / 2$. The profile of $\vartheta(x)$ consists of two domains, with domain walls of width $\lambda \ll L$ at $x_{ \pm}= \pm L / 4$ :

$$
\vartheta(x)= \begin{cases}\vartheta_{0} \tanh (x / \lambda-L / 4 \lambda) & \text { for } 0<x<L / 2, \\ -\vartheta_{0} \tanh (x / \lambda+L / 4 \lambda) & \text { for }-L / 2<x<0,\end{cases}
$$

see Fig. 1. As initial condition for the numerics we take a real Gaussian wave packet centered at $x=0$,

$$
\psi_{0}=\left(u_{0}, u_{0}\right), \quad u_{0}(x)=(2 \sigma \sqrt{\pi})^{-1 / 2} \exp \left(-x^{2} / 2 \sigma^{2}\right),
$$

normalized to unity, $\int \psi_{0}^{\dagger} \psi_{0} d x=1$. Figure 2 shows how this state collapses onto one of the two domain walls, depending on the sign of $\kappa$.

For the analytics we take the continuum limit of the discrete-time quantum walk, obtained from Eq. (5) under the assumption that the change $\delta \psi$ in one time step $\delta t$ is infinitesimal. The state-dependent rotation contributes a term $-i \delta t\left(\vartheta+\kappa \psi^{\dagger} \sigma_{z} \psi\right) \sigma_{y} \psi$ to $\delta \psi$, while the state-dependent shift contributes $-\delta t \sigma_{z} \partial \psi / \partial x$, resulting in the Dirac equation [2]

$$
i \frac{\partial \psi}{\partial t}=-i \sigma_{z} \frac{\partial \psi}{\partial x}+\left(\vartheta(x)+\kappa \psi^{\dagger} \sigma_{z} \psi\right) \sigma_{y} \psi .
$$

For large $L$ the two domain walls may be considered separately. The zero mode bound to the domain wall at $x_{ \pm}= \pm L / 4$ is

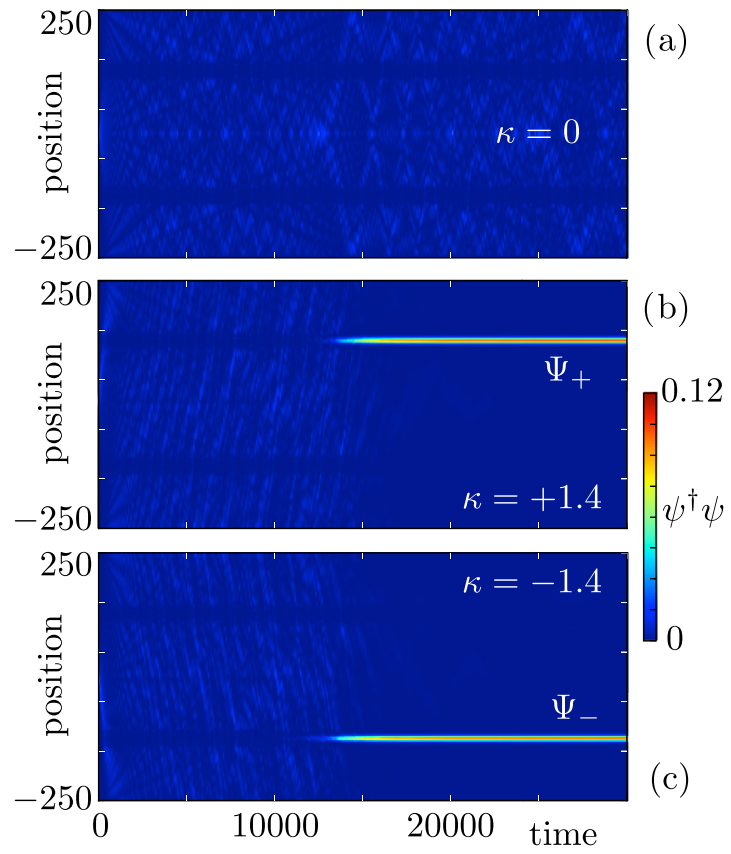

FIG. 2. Time evolution of the density $\psi_{t}^{\dagger} \psi_{t}$, starting from a real Gaussian wave packet $\psi_{0}=\left(u_{0}, u_{0}\right)$ [given by Eq. (7) with $\sigma^{2}=50$ ], for the quantum walk with rotation angle profile of Fig. 1. The three panels show the result for the linear quantum walk [panel (a), $\kappa=0$ ] and for the nonlinear quantum walk [panels (b) and (c), $\kappa= \pm 1.4$ ]. Depending on the sign of the nonlinearity, the state collapses onto the zero mode $\Psi_{+}$or $\Psi_{-}$.

given by

$$
\Psi_{ \pm} \propto\left(u_{ \pm}, \pm u_{ \pm}\right), \quad u_{ \pm}(x)=\exp \left( \pm \int_{x}^{0} \vartheta\left(x^{\prime}\right) d x^{\prime}\right) .
$$

The time-independent state $\Psi_{ \pm}$is an eigenvector of $\sigma_{x}$ with eigenvalue \pm 1 , selected by the sign of $\vartheta^{\prime}(x)$ at the domain wall.

We now perform a linear stability analysis for a real perturbation $\psi(x, t)=\Psi_{ \pm}(x)+\eta(x, t)$ of the zero mode. To linear order in $\eta$ we have

$$
\frac{\partial \eta}{\partial t}=-\sigma_{z} \frac{\partial \eta}{\partial x}-\vartheta(x) i \sigma_{y} \eta-2 \kappa u_{ \pm}^{2}(x)\left( \pm \eta-\sigma_{x} \eta\right) .
$$

We focus on perturbations $\eta=e^{i k x} \eta(t)$ of the zero mode with wave number $k \gtrsim 1 / \lambda$, so we may neglect the spatial dependence of $\vartheta(x)$ and $u_{ \pm}(x)$. The resulting ordinary differential equation,

$$
\frac{d \eta}{d t}=-\Gamma \eta, \quad \Gamma=i k \sigma_{z}+i \vartheta \sigma_{y}+2 \kappa u_{ \pm}^{2}\left( \pm 1-\sigma_{x}\right),
$$

has relaxation matrix $\Gamma$ with eigenvalues $\mu_{1}, \mu_{2}$ given by

$$
\begin{aligned}
& \mu_{1}= \pm 2 \kappa u_{ \pm}^{2}+\Delta, \quad \mu_{2}= \pm 2 \kappa u_{ \pm}^{2}-\Delta, \\
& \Delta^{2}=4 \kappa^{2} u_{ \pm}^{4}-k^{2}-\vartheta^{2} .
\end{aligned}
$$

We conclude that for $\kappa>0$ the zero mode $\Psi_{+}$is an attractor $\left(\operatorname{Re} \mu_{1}, \mu_{2}>0\right)$ and $\Psi_{-}$is a repeller $\left(\operatorname{Re} \mu_{1}, \mu_{2}<0\right)$, while for $\kappa<0$ the roles are interchanged. 


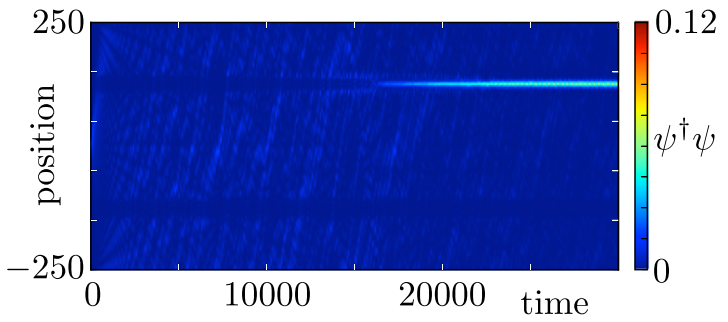

FIG. 3. Same as Fig. 2(b), but with a complex initial state $\psi_{0}=$ $\left(u_{0}, i u_{0}\right)$.

\section{INITIAL STATES WITHOUT PARTICLE-HOLE SYMMETRY}

Particle-hole symmetry ensures that a real $\psi$ remains real, but we might start with an initially complex state and ask for the stability of the zero mode under complex perturbations. Substitution into Eq. (8) of $\psi=\Psi_{ \pm}+\eta+i \zeta$, with real $\Psi_{ \pm}, \eta, \zeta$, shows that to first order in $\eta, \zeta$ the nonlinear term contains only the real perturbation:

$$
\begin{aligned}
\frac{\partial}{\partial t}(\eta+i \zeta)= & -\sigma_{z} \frac{\partial}{\partial x}(\eta+i \zeta)-\vartheta(x) i \sigma_{y}(\eta+i \zeta) \\
& -2 \kappa u_{ \pm}^{2}(x)\left( \pm \eta-\sigma_{x} \eta\right) .
\end{aligned}
$$

The relaxation matrix for the real perturbation is as in Eq. (11), with eigenvalues $\mu_{1}, \mu_{2}$ given by Eq. (12). But the relaxation matrix for the imaginary perturbation,

$$
\frac{d \zeta}{d t}=-\Gamma_{0} \zeta, \quad \Gamma_{0}=i k \sigma_{z}+i \vartheta \sigma_{y},
$$

has purely imaginary eigenvalues,

$$
\mu_{3}=i \sqrt{k^{2}+\vartheta^{2}}, \quad \mu_{4}=-i \sqrt{k^{2}+\vartheta^{2}} .
$$

More generally, a perturbation of a complex zero mode $\Psi_{ \pm}(x)=e^{i \phi}\left(u_{ \pm}, u_{ \pm}\right)$has (for $\kappa>0$ ) a decaying in-phase component $e^{i \phi} \eta$ and a nondecaying out-of-phase component $i e^{i \phi} \zeta$ [with real spinors $\eta=\left(\eta_{1}, \eta_{2}\right), \zeta=\left(\zeta_{1}, \zeta_{2}\right)$ ]. Figures 3 and 4 illustrate the resulting localized peak on the extended background.

\section{DISCUSSION}

Figure 2 summarizes our key finding: while the linear quantum walk is only slightly perturbed by the emergence of zero modes at a topological phase transition, once we turn on

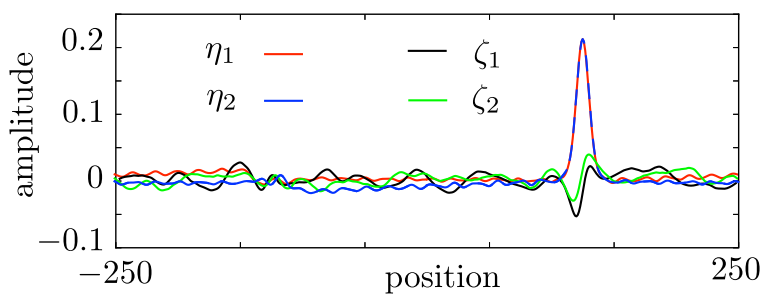

FIG. 4. Decomposition of the state $\psi=e^{i \phi}(\eta+i \zeta)$ at a late time $\left(t=8 \times 10^{4}\right)$, starting from the complex state $\psi_{0}=\left(u_{0}, u_{0}+i u_{0}\right)$, with $u_{0}$ the Gaussian wave packet $(7)(\kappa=1.4$, other parameters as in Fig. 1). The spinor $\eta=\left(\eta_{1}, \eta_{2}\right)$ is in phase with the zero mode $\Psi_{+}$; the spinor $\zeta=\left(\zeta_{1}, \zeta_{2}\right)$ is out of phase.

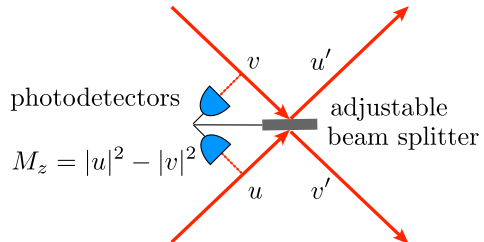

$u^{\prime}=\cos \left(\vartheta+\kappa M_{z}\right) u-\sin \left(\vartheta+\kappa M_{z}\right) v$
$v^{\prime}=\sin \left(\vartheta+\kappa M_{z}\right) u+\cos \left(\vartheta+\kappa M_{z}\right) v$

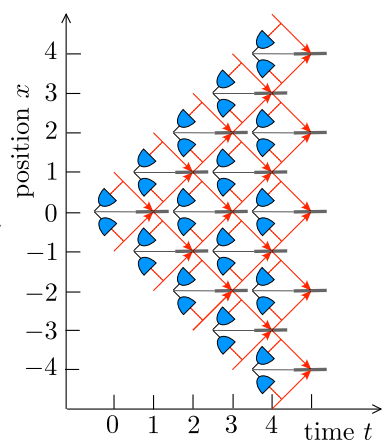

FIG. 5. Optical Galton board consisting of an array of beam splitters with an adjustable transmission, conditioned on the output of a pair of photodetectors. The left panel shows a single element of the array; the right panel shows their combination.

the nonlinearity the wave packet is steered towards a domain wall and trapped in a zero mode of definite chirality. This striking dynamics follows from a specific model calculation. How generic is it, and how might it be realized in an experiment?

For the experimental connection, we recall that quantum walks can be realized with true quantum mechanical elements [31] (ion traps, cold atoms, quantum dots)—or they can be simulated with classical waves [32,33], as in the optical Galton board [34-36]. Such a simulated quantum walk combines linear optical elements to mimic the quantum evolution of a spin-1/2 degree of freedom. Nonlinearities can be introduced via nonlinear optics [37], or while staying within linear optics by introducing a feedforward element conditioned on the output of a photodetector [38]. A scheme of the latter type [39] is illustrated in Fig. 5. This optical Galton board simulates a quantum walk with evolution operator $S R_{\vartheta} \exp \left(-i \kappa M_{z} \sigma_{y}\right)$, which differs from Eqs. (3) and (5) by the order of the operators $\left(S R_{\vartheta}\right.$ instead of $\left.R_{\vartheta / 2} S R_{\vartheta / 2}\right)$. In the continuum limit of Eq. (8) this order is irrelevant, and we have checked numerically that the dynamics is essentially the same as in Fig. 2.

Concerning the generality of the result, we have two necessary conditions for the nonlinearity: it should preserve the zero mode as a fixed point of the dynamics and it should contract phase space, breaking the area preservation of the linear dynamics. Both conditions hold if Eq. (5) is replaced by

$$
\begin{aligned}
\psi_{t+1} & =U \bar{\psi}_{t}, \quad \bar{\psi}_{t}=\exp (-i \tilde{\kappa} \tilde{M} \hat{n} \cdot \hat{\sigma}) \psi_{t}, \\
\tilde{M} & =\psi_{t}^{\dagger}(\hat{m} \cdot \hat{\sigma}) \psi_{t},
\end{aligned}
$$

with $\hat{\sigma}=\left(\sigma_{x}, \sigma_{y}, \sigma_{z}\right)$ and two unit vectors $\hat{n}=\left(0, n_{y}, n_{z}\right)$ and $\hat{m}=\left(0, m_{y}, m_{z}\right)$ satisfying $\hat{m} \times \hat{n} \neq 0$ (otherwise the map would be area preserving). Particle-hole symmetry is broken for $n_{z} \neq 0$, but the zero mode $\Psi_{ \pm}$is preserved. A complex perturbation $\delta \psi$ has relaxation matrix $d \delta \psi=-\tilde{\Gamma} \delta \psi$ with eigenvalues $\tilde{\mu}_{n}, n=1,2,3,4$, given by Eqs. (12) and (15), upon the replacement $\kappa \mapsto \tilde{\kappa}(\hat{n} \times \hat{m}) \cdot \hat{x}$. The attractor-repeller pair is preserved, demonstrating the generality of our findings.

We finally note that discrete time quantum walks have been used as a design principle for quantum algorithms. For instance, the search algorithms of Refs. [40,41] can be understood in terms of bound states in effectively one-dimensional 
quantum walks. The key observations in this paper, namely the convergence towards certain bound states from arbitrary initial states, as well as the accelerated escape from unwanted bound states, thus may have promising implications for quantum algorithms. This is in line with several other recent results on continuous time quantum walks, where nonlinearities are observed to speed up quantum algorithms [26].

\section{ACKNOWLEDGMENTS}

We acknowledge discussions on the optical implementation with W. Löffler. This research was supported by the Foundation for Fundamental Research on Matter (FOM), the Netherlands Organization for Scientific Research (NWO/OCW), and an ERC Synergy Grant.
[1] Y. Aharonov, L. Davidovich, and N. Zagury, Phys. Rev. A 48, 1687 (1993).

[2] D. A. Meyer, J. Stat. Phys. 85, 551 (1996).

[3] E. Farhi and S. Gutmann, Phys. Rev. A 58, 915 (1998).

[4] J. Kempe, Contemp. Phys. 44, 307 (2003).

[5] S. E. Venegas-Andraca, Quant. Inf. Proc. 11, 1015 (2012).

[6] A. Joye and M. Merkli, J. Stat. Phys. 140, 1025 (2010).

[7] A. Ahlbrecht, H. Vogts, A. H. Werner, and R. F. Werner, J. Math. Phys. 52, 042201 (2011).

[8] A. Ahlbrecht, V. B. Scholz, and A. H. Werner, J. Math. Phys. 52, 102201 (2011).

[9] A. Schreiber, K. N. Cassemiro, V. Potoček, A. Gábris, I. Jex, and Ch. Silberhorn, Phys. Rev. Lett. 106, 180403 (2011).

[10] H. Obuse and N. Kawakami, Phys. Rev. B 84, 195139 (2011).

[11] J. Ghosh, Phys. Rev. A 89, 022309 (2014).

[12] J. M. Edge and J. K. Asboth, Phys. Rev. B 91, 104202 (2015).

[13] M. Karski, L. Förster, J.-M. Choi, A. Steffen, W. Alt, D. Meschede, and A. Widera, Science 325, 174 (2009).

[14] M. S. Rudner and L. S. Levitov, Phys. Rev. Lett. 102, 065703 (2009).

[15] F. Zähringer, G. Kirchmair, R. Gerritsma, E. Solano, R. Blatt, and C. F. Roos, Phys. Rev. Lett. 104, 100503 (2010).

[16] T. Kitagawa, M. S. Rudner, E. Berg, and E. Demler, Phys. Rev. A 82, 033429 (2010).

[17] T. Kitagawa, M. A. Broome, A. Fedrizzi, M. S. Rudner, E. Berg, I. Kassal, A. Aspuru-Guzik, E. Demler, and A. G. White, Nat. Commun. 3, 882 (2012).

[18] J. K. Asboth, B. Tarasinski, and P. Delplace, Phys. Rev. B 90, 125143 (2014).

[19] F. Cardano, M. Maffei, F. Massa, B. Piccirillo, C. De Lisio, G. De Filippis, V. Cataudella, E. Santamato, and L. Marrucci, arXiv:1507.01785.

[20] C. Poli, M. Bellec, U. Kuhl, F. Mortessagne, and H. Schomerus, Nat. Commun. 6, 6710 (2015)

[21] J. M. Zeuner, M. C. Rechtsman, Y. Plotnik, Y. Lumer, S. Nolte, M. S. Rudner, M. Segev, and A. Szameit, Phys. Rev. Lett. 115, 040402 (2015).

[22] Y. Lahini, M. Verbin, S. D. Huber, Y. Bromberg, R. Pugatch, and Y. Silberberg, Phys. Rev. A 86, 011603(R) (2012).

[23] C.-W. Lee, P. Kurzyński, and H. Nha, Phys. Rev. A 92, 052336 (2015).

[24] C. Navarrete-Benlloch, A. Pérez, and E. Roldán, Phys. Rev. A 75, 062333 (2007).
[25] G. Di Molfetta, F. Debbasch, and M. Brachet, Phys. Rev. E 92, 042923 (2015).

[26] D. A. Meyer and T. G. Wong, Phys. Rev. A 89, 012312 (2014).

[27] W. P. Su, J. R. Schrieffer, and A. J. Heeger, Phys. Rev. Lett. 42, 1698 (1979).

[28] J. K. Asboth and H. Obuse, Phys. Rev. B 88, 121406 (2013).

[29] In addition to the zero mode with $U \Psi=\Psi$, the domain wall may also support a bound state with $U \Psi=-\Psi$. Because this state is rapidly oscillating on the scale of the lattice constant, it plays no role in the long-wavelength dynamics considered here.

[30] The fact that the zero mode is an eigenstate of $\sigma_{x}$ follows from $U \Psi=\Psi$ and $U \sigma_{x} \Psi=\sigma_{x}\left(\sigma_{x} U\right)^{2} U^{-1} \Psi=\sigma_{x} \Psi$. Since the zero mode is nondegenerate, the two states $\Psi$ and $\sigma_{x} \Psi$ must be linearly related.

[31] K. Manouchehri and J. Wang, Physical Implementation of Quantum Walks (Springer, Berlin, 2014).

[32] P. L. Knight, E. Roldan, and J. E. Sipe, Phys. Rev. A 68, 020301 (2003).

[33] H. Jeong, M. Paternostro, and M. S. Kim, Phys. Rev. A 69, 012310 (2004).

[34] D. Bouwmeester, I. Marzoli, G. P. Karman, W. Schleich, and J. P. Woerdman, Phys. Rev. A 61, 013410 (1999).

[35] B. Do, M. L. Stohler, S. Balasubramanian, D. S. Elliott, C. Eash, E. Fischbach, M. A. Fischbach, A. Mills, and B. Zwickl, J. Opt. Soc. Am. B 22, 499 (2005).

[36] A. Schreiber, K. N. Cassemiro, V. Potoček, A. Gabris, P. J. Mosley, E. Andersson, I. Jex, and Ch. Silberhorn, Phys. Rev. Lett. 104, 050502 (2010).

[37] A. S. Solntsev, A. A. Sukhorukov, D. N. Neshev, and Y. S. Kivshar, Phys. Rev. Lett. 108, 023601 (2012).

[38] Y. Shikano, T. Wada, and J. Horikawa, Sci. Rep. 4, 4427 (2014).

[39] In the implementation of an optical Galton board shown in Fig. 5, the photon polarization plays no role and the spin- $1 / 2$ degree of freedom of the quantum walk is fully orbital [18]. The adjustable beam splitter combines the rotation and shift operators $R_{\vartheta}$ and $S$ in a single step. Alternative split-step implementations can use adjustable polarizers for $R_{\vartheta}$, followed by polarizing beam splitters [35] or birefringent displacers [17] for $S$.

[40] N. Shenvi, J. Kempe, and K. Birgitta Whaley, Phys. Rev. A 67, 052307 (2003).

[41] A. Ambainis, J. Kempe, and A. Rivosh, in Proceedings of the Sixteenth Annual ACM-SIAM Symposium on Discrete Algorithms (SIAM, Philadelphia, 2005), pp. 1099-1108. 